\title{
A MEASUREMENT OF THE CORRELATION OF GALAXY SURVEYS WITH CMB LENSING CONVERGENCE MAPS FROM THE SOUTH POLE TELESCOPE
}

L. E. Bleem ${ }^{1,2}$, A. van Engelen ${ }^{3}$, G. P. Holder $^{3}$, K. A. Aird ${ }^{4}$, R. Armstrong ${ }^{5}$, M. L. N. Ashby ${ }^{6}$, M. R. Becker ${ }^{1,2}$, B. A. Benson ${ }^{1,7}$, T. Biesiadzinski ${ }^{8}$, M. Brodwin ${ }^{9}$, M. T. Busha ${ }^{10,11}$, J. E. Carlstrom ${ }^{1,2,7,12,13}$, C. L. ChanG ${ }^{1,7,13}$, H. M. ChO $^{14}$, T. M. Crawford ${ }^{1,12}$, A. T. Crites ${ }^{1,12}$, T. de HaAn ${ }^{3}$, S. Desai $^{15,16}$, M. A. Dobbs ${ }^{3}$, O. Doré $^{17,18}$ J. Dudley $^{3}$, J. E. Geach ${ }^{3}$, E. M. George ${ }^{19}$, M. D. Gladders ${ }^{1,12}$, A. H. Gonzalez ${ }^{20}$, N. W. Halverson ${ }^{21}$, N. Harrington ${ }^{19}$, F. W. High ${ }^{1,12}$, B. P. Holden ${ }^{22}$, W. L. Holzapfel ${ }^{19}$, S. Hoover ${ }^{1,2}$, J. D. Hrubes ${ }^{4}$, M. JoY ${ }^{23}$, R. Keisler ${ }^{1,2}$, L. KNOX $^{24}$, A. T. LeE $^{19,11}$, E. M. Leitch ${ }^{1,12}$, M. Lueker ${ }^{17,19}$, D. Luong-VAN ${ }^{4}$, D. P. Marrone ${ }^{25}$, J. Martinez-Manso ${ }^{20}$, J. J. McMahon $^{8}$, J. Mehl $^{1}$, S. S. Meyer ${ }^{1,2,7,12}$, J. J. Mohr ${ }^{15,16,26}$, T. E. Montroy ${ }^{27}$, T. Natoli ${ }^{1,2}$, S. Padin ${ }^{1,12,17}$, T. PlagGe $^{1,12}$, C. Pryke $^{28}$, C. L. Reichardt ${ }^{19}$, A. Rest ${ }^{29}$, J. E. Ruhl ${ }^{27}$, B. R. SaliwanchiK ${ }^{27}$, J. T. SAYre ${ }^{27}$, K. K. Schaffer ${ }^{1,7,30}$, L. Shaw $^{31}$, E. ShirokofF ${ }^{19}$, H. G. Spieler ${ }^{11}$, B. Stalder ${ }^{6}$, S. A. Stanford ${ }^{24}$, Z. Staniszewski ${ }^{27}$, A. A. Stark ${ }^{6}$, D. Stern ${ }^{18}$, K. Story ${ }^{1,2}$,

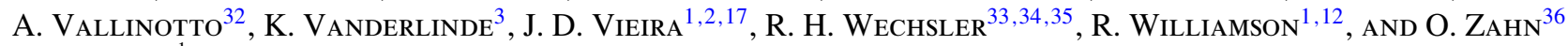

${ }^{1}$ Kavli Institute for Cosmological Physics, University of Chicago, 5640 South Ellis Avenue, Chicago, IL 60637, USA

${ }^{2}$ Department of Physics, University of Chicago, 5640 South Ellis Avenue, Chicago, IL 60637, USA

${ }^{3}$ Department of Physics, McGill University, 3600 Rue University, Montreal, Quebec H3A 2T8, Canada ${ }^{4}$ University of Chicago, 5640 South Ellis Avenue, Chicago, IL 60637, USA

${ }^{5}$ National Center for Supercomputing Applications, University of Illinois, 1205 West Clark Street, Urbana, IL 61801, USA

${ }^{6}$ Harvard-Smithsonian Center for Astrophysics, 60 Garden Street, Cambridge, MA 02138, USA

${ }^{7}$ Enrico Fermi Institute, University of Chicago, 5640 South Ellis Avenue, Chicago, IL 60637, USA

${ }^{8}$ Department of Physics, University of Michigan, 450 Church Street, Ann Arbor, MI 48109, USA

${ }^{9}$ Department of Physics and Astronomy, University of Missouri, 5110 Rockhill Road, Kansas City, MO 64110, USA

${ }^{10}$ Institute for Theoretical Physics, University of Zürich, Zürich, Switzerland

${ }^{11}$ Physics Division, Lawrence Berkeley National Laboratory, Berkeley, CA 94720, USA

${ }^{12}$ Department of Astronomy and Astrophysics, University of Chicago, 5640 South Ellis Avenue, Chicago, IL 60637, USA

${ }^{13}$ Argonne National Laboratory, 9700 South Cass Avenue, Argonne, IL 60439, USA

${ }^{14}$ NIST Quantum Devices Group, 325 Broadway, Mailcode 817.03, Boulder, CO 80305, USA

${ }^{15}$ Department of Physics, Ludwig-Maximilians-Universität, Scheinerstr. 1, 81679 München, Germany ${ }^{16}$ Excellence Cluster Universe, Boltzmannstr. 2, 85748 Garching, Germany

${ }^{17}$ Division of Physics, Mathematics, and Astronomy, California Institute of Technology, MS 249-17, 1216 East California Blvd., Pasadena, CA 91125, USA

18 Jet Propulsion Laboratory, California Institute of Technology, Pasadena, CA 91109, USA

${ }_{19}^{19}$ Department of Physics, University of California, Berkeley, CA 94720, USA

${ }^{20}$ Department of Astronomy, University of Florida, Gainesville, FL 32611, USA

${ }^{21}$ Department of Astrophysical and Planetary Sciences and Department of Physics, University of Colorado, Boulder, CO 80309, USA

${ }^{22}$ SantaCruz UCO/Lick Observatories, University of California, Santa Cruz, CA 95065, USA

${ }^{23}$ Department of Space Science, VP62, NASA Marshall Space Flight Center, Huntsville, AL 35812, USA

${ }^{24}$ Department of Physics, University of California, One Shields Avenue, Davis, CA 95616, USA

${ }^{25}$ Steward Observatory, University of Arizona, 933 North Cherry Avenue, Tucson, AZ 85721, USA

${ }^{26}$ Max-Planck-Institut für extraterrestrische Physik, Giessenbachstr. 85748 Garching, Germany

${ }^{27}$ Physics Department, Center for Education and Research in Cosmology and Astrophysics, Case Western Reserve University, Cleveland, OH 44106, USA

${ }^{28}$ Department of Physics, University of Minnesota, 116 Church Street Southeast, Minneapolis, MN 55455, USA

${ }^{29}$ Space Telescope Science Institute, 3700 San Martin Drive, Baltimore, MD 21218, USA

${ }^{30}$ Liberal Arts Department, School of the Art Institute of Chicago, 112 South Michigan Avenue, Chicago, IL 60603, USA

${ }^{31}$ Department of Physics, Yale University, P.O. Box 208210, New Haven, CT 06520-8120, USA

${ }^{32}$ T-2, MS B285, Los Alamos National Laboratory, Los Alamos, NM 87545, USA

${ }^{33}$ Kavli Institute for Particle Astrophysics and Cosmology, 452 Lomita Mall, Stanford University, Stanford, CA 94305, USA

${ }^{34}$ Department of Physics, Stanford University, Stanford, CA 94305, USA

${ }^{35}$ SLAC National Accelerator Laboratory, 2575 Sand Hill Road, MS 29, Menlo Park, CA 94025, USA

${ }^{36}$ Berkeley Center for Cosmological Physics, Department of Physics, University of California, and Lawrence Berkeley National Labs, Berkeley, CA 94720, USA

Received 2012 March 21; accepted 2012 May 29; published 2012 June 13

\section{ABSTRACT}

We compare cosmic microwave background lensing convergence maps derived from South Pole Telescope (SPT) data with galaxy survey data from the Blanco Cosmology Survey, WISE, and a new large Spitzer/IRAC field designed to overlap with the SPT survey. Using optical and infrared catalogs covering between 17 and 68 deg $^{2}$ of sky, we detect a correlation between the SPT convergence maps and each of the galaxy density maps at $>4 \sigma$, with zero correlation robustly ruled out in all cases. The amplitude and shape of the cross-power spectra are in good agreement with theoretical expectations and the measured galaxy bias is consistent with previous work. The detections reported here utilize a small fraction of the full $2500 \mathrm{deg}^{2}$ SPT survey data and serve as both a proof of principle of the technique and an illustration of the potential of this emerging cosmological probe.

Key words: cosmic background radiation - galaxies: structure

Online-only material: color figures 


\section{INTRODUCTION}

Gravitational lensing of the primordial anisotropies of the cosmic microwave background (CMB) imprints information about the density fluctuations between $z \sim 1100$ and the present day onto the observed CMB fluctuations. This information can be extracted by measuring the induced correlation between initially independent spatial modes of the CMB and used to construct a map of the lensing convergence field. This field is closely related to the projected gravitational potential.

The statistics of CMB lensing maps provide powerful constraints on cosmological parameters (Lesgourgues et al. 2006; de Putter et al. 2009). Such maps can also be combined with other tracers of large-scale structure to test cosmological models and constrain properties of the tracer population. One such analysis involves cross-correlating lensing maps with galaxy catalogs. The correlation measures the average lensing signal from the dark matter halos that host the galaxies and can be used to determine the halo bias. These measurements test models of the time evolution of cosmic density fluctuations and of primordial non-Gaussianity (Dalal et al. 2008; Jeong et al. 2009).

CMB lensing is a young field. The first detections-using lensing-galaxy cross-correlations-were reported relatively recently (Smith et al. 2007; Hirata et al. 2008). The first detections of lensing in CMB data alone have come from high-resolution CMB experiments, both through non-Gaussianity (Das et al. 2011b; van Engelen et al. 2012) and through smearing of the acoustic peaks (Reichardt et al. 2009; Das et al. 2011a; Keisler et al. 2011). Within the next year, further advances are expected: the Planck satellite (The Planck Collaboration 2006) will create all-sky convergence maps (Hanson et al. 2011), and the now-completed $2500 \mathrm{deg}^{2}$ South Pole Telescope (SPT) survey (Carlstrom et al. 2011) will produce complementary lensing maps that have significantly higher signal-to-noise per mode than the Planck maps.

Here, we present the results of the cross-correlation of several galaxy populations with convergence maps from the SPT. In contrast to previous detections of this cross-correlation that utilized a large fraction of the sky with low signal-to-noise lensing maps (Smith et al. 2007; Hirata et al. 2008), we use convergence maps that have signal-to-noise greater than 1 on degree scales but significantly less sky area; the results presented are from two $\sim 100 \mathrm{deg}^{2}$ SPT fields. A significant cross-correlation is detected between the convergence maps and maps of galaxy density constructed from optical and infrared (IR) catalogs in each of the two fields.

The Letter is structured as follows. We first discuss the underlying theory and provide a brief overview of the process of making convergence maps from CMB data. We next describe the catalogs, real and simulated, that we cross-correlate with the lensing maps. We conclude with a discussion of the results and the potential of upcoming large CMB lensing data sets and large-area galaxy surveys.

\section{THEORY}

The CMB lensing convergence in a direction $\hat{\mathbf{n}}$ on the sky is given in terms of the matter fluctuations by a line-of-sight integration,

$$
\kappa(\hat{\mathbf{n}})=\int d \chi W^{\kappa}(\chi) \delta(\chi \hat{\mathbf{n}}, z(\chi)),
$$

where $\delta(\mathbf{r}, z)$ is the fractional matter overdensity at comoving position $\mathbf{r}$ and redshift $z$, and the distance kernel is (Cooray \&
Hu 2000; Song et al. 2003)

$$
W^{\kappa}(\chi)=\frac{3}{2} \Omega_{m} H_{0}^{2} \frac{\chi}{a(\chi)} \frac{\chi_{\mathrm{CMB}}-\chi}{\chi_{\mathrm{CMB}}} .
$$

Here, $\Omega_{m}$ is the matter density relative to the critical density evaluated today, $H_{0}$ is the Hubble parameter today, $a(\chi)$ is the cosmological scale factor, $\chi_{\mathrm{CMB}} \simeq 14 \mathrm{Gpc}$ is the comoving distance to the CMB recombination surface, and we have assumed a spatially flat universe.

Under the assumption that observed galaxies are biased tracers of mass fluctuations, the observed fractional galaxy overdensity in a direction $\hat{\mathbf{n}}$ is given by

$$
g(\hat{\mathbf{n}})=\int d \chi W^{g}(\chi) \delta(\chi \hat{\mathbf{n}}, z(\chi)),
$$

where the distance kernel is

$$
W^{g}(\chi)=\frac{1}{\left[\int d z^{\prime} \frac{d N\left(z^{\prime}\right)}{d z^{\prime}}\right]} \frac{d z}{d \chi} \frac{d N(z)}{d z} b(\chi) .
$$

Here, $d N(z) / d z$ is the distribution of galaxies in redshift and $b(\chi)$ is the bias of the galaxies relative to the dark matter density, assumed here to be independent of scale.

The cross power between the convergence and the galaxy overdensity at a multipole $L$ is given in the Limber approximation (Limber 1953; Kaiser 1992) by

$$
C_{L}^{\kappa g}=\int d z \frac{d \chi}{d z} \frac{1}{\chi^{2}} W^{\kappa}(\chi) W^{g}(\chi) P\left(k=\frac{L}{\chi}, z\right),
$$

where the matter power spectrum today, $P(k, 0)$, is given by $\left\langle\delta^{\star}(\mathbf{k}) \delta\left(\mathbf{k}^{\prime}\right)\right\rangle=(2 \pi)^{3} \delta_{\text {Dirac }}\left(\mathbf{k}-\mathbf{k}^{\prime}\right) P(k, 0)$ under the assumption of independent Fourier modes $\mathbf{k}$ and $\mathbf{k}^{\prime}$. The amount of cross-correlation is thus determined by the overlap between the two kernels, weighted by the matter power spectrum. The factors which determine this overlap, namely, $(1 / \chi) W^{[g, \kappa]}(z)(P(k, z) d \chi / d z)^{1 / 2}$, are plotted for the CMB lensing convergence and several galaxy populations in the bottom panel of Figure 1.

\section{CONVERGENCE MAPS FROM CMB LENSING}

CMB maps of two fields, one centered at (R.A., decl.) = $\left(23^{\mathrm{h}} 30^{\mathrm{m}},-55^{\mathrm{d}}\right)$ and the other at $\left(5^{\mathrm{h}} 30^{\mathrm{m}},-55^{\mathrm{d}}\right)$, were constructed using $150 \mathrm{GHz}$ data from the SPT survey. These fields together encompass $185 \mathrm{deg}^{2}$ and are the deepest SPT fields to date as they were observed with roughly twice the time per unit area as the rest of the SPT survey. In addition to the 2008 data used in van Engelen et al. (2012, hereafter V12), the maps used in this analysis include data from the 2010-2011 observing seasons. As such, the resulting lensing maps are $\sim 10 \%$ lower in noise than the typical maps to be expected from the rest of the survey.

Convergence maps were constructed using a quadratic estimator (Hu \& Okamoto 2002), as outlined in V12. Point sources with signal-to-noise greater than 6 were masked, while clusters detected via the thermal Sunyaev-Zeldovich (tSZ) effect were masked if they had a signal-to-noise greater than 6 in the 2008 data alone. This cross-correlation study is less sensitive to foreground contamination and offsets in power than the V12 analysis; therefore, the temperature maps were filtered to include modes from $l=1200$ to 4000, a larger range than the $l=$ 1200-3000 used in V12. 


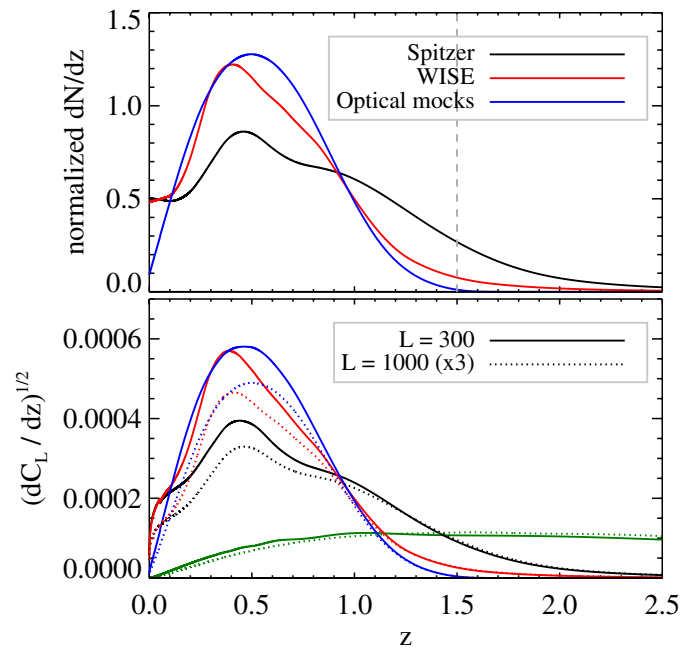

Figure 1. Redshift distribution for the galaxy samples are shown in the top panel. Mock catalogs (Section 5) are used to estimate the distribution for the optically selected galaxies, while IR-selected galaxy distributions are based on photometric redshift estimates from Brodwin et al. (2006). The mock catalogs extend to $z=1.3$, a sufficient redshift limit for the optical catalogs under consideration. The vertical dashed line at $z=1.5$ indicates the maximum redshift of the Brodwin et al. (2006) spectroscopic training sample. The quantities shown in the bottom panel, which includes the CMB lensing power (green lines), are the curves that one would multiply to obtain $d C_{L}^{\kappa g} / d z$, the integrand of Equation (5), at $L=300$ (solid curves) and $L=1000$ (dotted curves), assuming galaxy bias $b(z)=1$. The dotted curves have been multiplied by 3 for clarity.

(A color version of this figure is available in the online journal.)

The effect of foreground contamination is expected to be small for this analysis. Residual foreground emission in the $\mathrm{CMB}$ map that is correlated with the galaxy distribution is likely to lead to negative cross-correlations on large scales: excess small-scale power fluctuations in the temperature map are identified by the quadratic estimator as large-scale underdense regions which "squeeze" the sky. This produces an anticorrelation of true large-scale structure with residual foreground contamination in the lensing reconstruction. Conversely, dust in our Galaxy could also be interpreted by the lensing estimator as large-scale underdense regions while also suppressing the galaxy counts, leading to a net positive correlation. We discuss tests for foreground contamination in Section 6.

The measured CMB lensing signal was calibrated with simulations. As outlined in V12, simulated lensed CMB skies including contributions from faint IR sources and tSZ from lowmass galaxy clusters (both modeled as Gaussian random fields) were added to noise generated from SPT time streams. The simulations were filtered and analyzed in the same way as the real data. We normalize the reconstructed convergence maps as a function of $L$ by comparing the cross-spectra of the input and output maps to the power spectra of the input maps. The simulated lensing maps are also used to estimate the uncertainty in the cross-correlation.

\section{OPTICAL- AND IR-SELECTED CATALOGS}

We use optical- and IR-selected catalogs to create maps of the fractional galaxy overdensity, $(N-\bar{N}) / \bar{N}$, where $N$ is the galaxy counts in a $1^{\prime}$ cell. A bright magnitude threshold is applied to each catalog to reduce contamination from Galactic objects, while a faint magnitude limit is set to ensure uniformity in source detection. We exclude regions around bright stars found in the Two Micron All Sky Survey source catalog (Skrutskie et al. 2006).

Optical catalogs were produced using data from the Blanco Cosmology Survey (BCS; Desai et al. 2012). The survey consists of two fields, a $5 \mathrm{~h}$ and a $23 \mathrm{~h}$ field, both contained within the SPT footprint. For this analysis, we extracted sources from images reduced using the pipeline described in Rest et al. (2005) and Miknaitis et al. (2007). Source detection was performed using SExtractor v2.8.6 (Bertin \& Arnouts 1996), and the photometry was calibrated using Stellar Locus Regression (High et al. 2009). We restrict the analysis to areas that are complete to at least $i_{\mathrm{AB}}=22.5$ and construct catalogs from all sources $19.5<i_{\mathrm{AB}}<22.5$.

In the $23 \mathrm{~h}$ field, we also use data from the SPT Spitzer Deep Field (M. L. N. Ashby 2012, private communication), an ongoing survey of $100 \mathrm{deg}^{2}$ at 3.6 and $4.5 \mu \mathrm{m}$. Data reduction followed the methods of Ashby et al. (2009), except, owing to the new survey's large size, photometry from individual $2 \mathrm{deg}^{2}$ subfields was merged in catalog space instead of extracted from a single monolithic mosaic. Here, we analyze $32 \mathrm{deg}^{2}$ of existing coverage and include all sources with $4.5 \mu \mathrm{m}$ magnitude between 15 and 17 Vega.

As a second catalog in the $5 \mathrm{~h}$ field we use the sources from the WISE (Wright et al. 2010) all-sky data release. We restrict this analysis to areas with W1 band exposure times between 231 and $616 \mathrm{~s}$, exclude regions with potentially corrupted objects as identified by the WISE pipeline, and include all sources with W1 magnitude between 15 and 17 Vega. Although the WISE selection function is complicated at these magnitudes (owing to source confusion ${ }^{37}$ and spatial variability in the imaging depth), we find it necessary to use these faint sources to obtain sufficient numbers of high-redshift galaxies. Despite its limitations, the WISE sample is a particularly interesting test case, as WISE is an all-sky survey, and this analysis will soon be possible over the entire SPT survey area.

The expected redshift distributions for the galaxy catalogs are shown in Figure 1. Summary statistics are presented in Table 1, and galaxy density maps are shown in Figure 2.

\section{MOCK CATALOGS}

Mock catalogs were used to estimate both the expected level of cross-correlation between $\mathrm{CMB}$ convergence maps and galaxy catalogs and the potential contamination from tSZ. The simulated galaxy sample is drawn from a $220 \mathrm{deg}^{2}$ lightcone populated with galaxies in the redshift range $0-1.3$. The underlying dark matter distribution is based on a cosmological simulation of $1 \mathrm{~h}^{-1} \mathrm{Gpc}$; this simulation is a single "Carmen" simulation from the Large Suite of Dark Matter Simulations project (see http://lss.phy.vanderbilt.edu/lasdamas/). The Adding Density Determined GAlaxies to Lightcone Simulations (ADDGALS) algorithm is run to assign galaxies to the dark matter particles in a way that reproduces the known luminosities, colors, and clustering properties of galaxies. The simulated catalogs have previously been used for tests of cluster finding (Koester et al. 2007), photometric redshifts (Gerdes et al. 2010), and spectroscopic follow-up (Cunha et al. 2012). A full description of the algorithm and the simulated sky catalog will be presented in Wechsler et al. and M. T. Busha et al. (in preparation). The simulated CMB lensing map based on the same underlying dark matter distribution was produced using multiple-plane raytracing methods similar to those of Hilbert et al. (2009), with

\footnotetext{
37 http://wise2.ipac.caltech.edu/docs/release/allsky/expsup/sec6_5.html
} 
Table 1

Field Parameters and Correlation Statistics

\begin{tabular}{lccccrr}
\hline \hline Field & $\begin{array}{c}\text { Area } \\
\left(\mathrm{deg}^{2}\right)\end{array}$ & $\begin{array}{c}\text { Density } \\
\left(\frac{\text { sources }}{\mathrm{deg}^{2}}\right)\end{array}$ & $\begin{array}{c}A \\
\left(C_{L} \times 10^{-7}\right)\end{array}$ & $n$ & $\begin{array}{c}\chi^{2} \\
(B e s t \text { fit })\end{array}$ & $\Delta \chi^{2}(0)$ \\
\hline WISE (5h) & 68.1 & $6.9 \times 10^{3}$ & $0.19 \pm 0.05$ & $-1.2 \pm 0.3$ & 8.8 & 19.6 \\
BCS (5h) & 27.0 & $2.5 \times 10^{4}$ & $0.27 \pm 0.06$ & $-1.8 \pm 0.3$ & 11.3 & 23.5 \\
BCS (23h) & 16.9 & $2.35 \times 10^{4}$ & $0.24 \pm 0.07$ & $-1.7 \pm 0.3$ & 9.6 & $1.2 \pm 0.3$ \\
Spitzer (23h) & 29.8 & $1.4 \times 10^{4}$ & $0.33 \pm 0.07$ & $-1.6 \pm 0.2$ & 13.7 & 28.9 \\
\hline
\end{tabular}

Notes. Galaxy catalog properties and results of power-law fits to lensing-galaxy cross-spectra. For each catalog, we report the best-fit amplitude and power-law index, $\chi^{2}$ of the best fit, the difference in $\chi^{2}$ from best fit to a model with zero cross-correlation, and the bias. Note: we report the weighted area of the galaxy density maps multiplied by the lensing apodization mask.
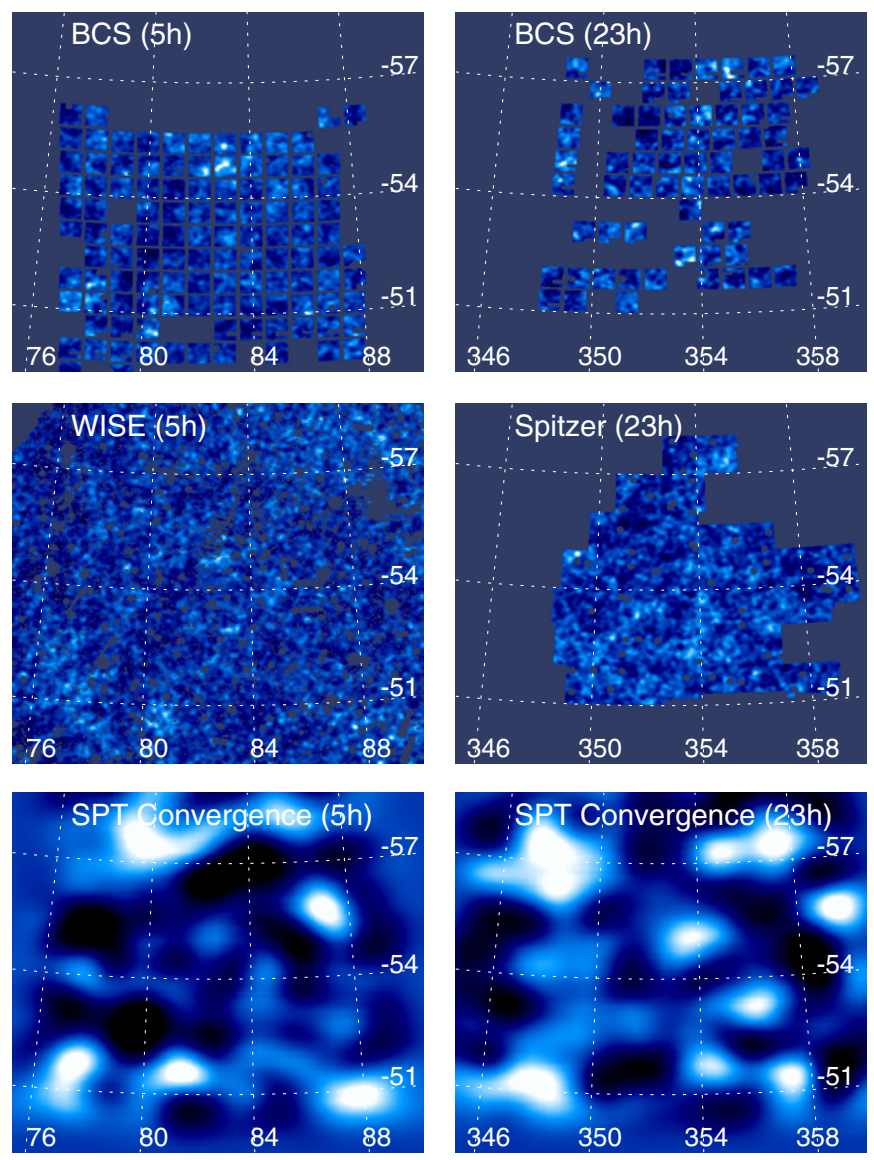

Figure 2. Top four panels show fractional galaxy overdensity maps smoothed to $6^{\prime}$ resolution. The bottom two panels show the lensing maps smoothed to $1^{\circ}$ resolution. At this scale, the signal-to-noise in the lensing maps is slightly greater than 1. While the lensing maps shown here have been smoothed to highlight real mass fluctuations, it is clear from the cross-spectra there is substantial information at smaller scales as well. The color scale is $\pm 80 \%$ of the maximum deviation from the mean in each map.

(A color version of this figure is available in the online journal.)

modifications to produce maps with the proper lensing kernel at the redshift of the CMB. Thermal SZ was included in the simulation as in Biesiadzinski et al. (2012): tSZ profiles were generated at the location of the halos using the Arnaud et al. (2010) model and projected along the line of sight to create a simulated tSZ map.

Owing to the finite size of the simulation box, the mock catalogs and the CMB lensing map only extend to $z \sim 1.3$. For optical catalogs, this is not a serious limitation but there could be substantial cross-correlation coming from higher redshifts

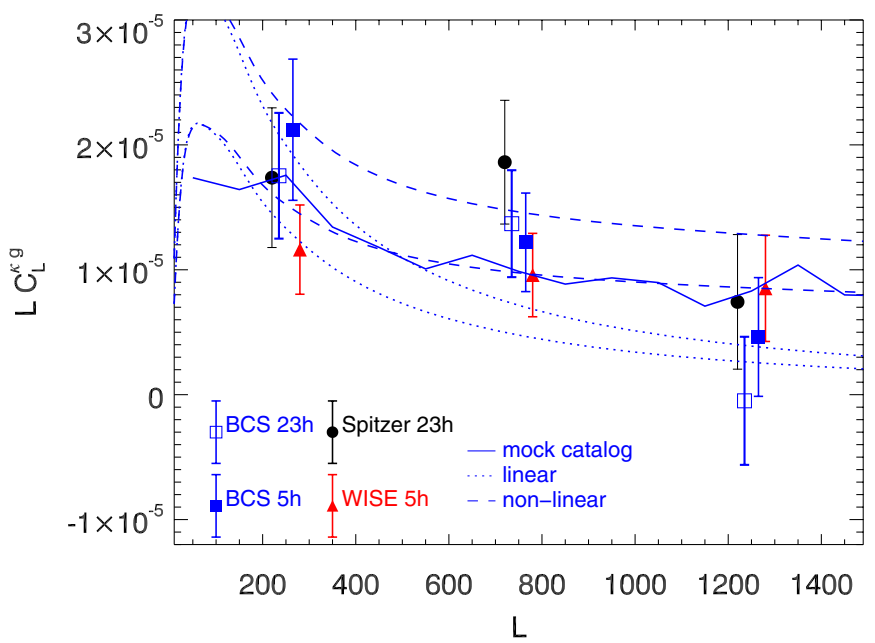

Figure 3. Cross-spectra of galaxy number density maps with SPT lensing convergence maps. Spectra are calculated in 14 bins from $L=150-1450$, but are shown combined into 3 bins for display purposes. The solid blue line is calculated using the mock catalogs and convergence maps. The dashed and dotted lines are obtained using the Limber approximation and the redshift distribution from the mock catalogs for the nonlinear and linear power spectrum, respectively. The upper and lower curves for each model correspond to a redshiftindependent galaxy bias of 1.0 and 1.5 , respectively. For clarity we only plot models for the optical catalogs. Predictions for the IR samples are within $15 \%$ of the plotted curves.

(A color version of this figure is available in the online journal.)

in the IR-selected catalogs. Also, these mock catalogs do not include active galactic nuclei (which could enhance the crosscorrelation), stars (which would not be correlated with a CMB lensing map), or the effects of source confusion (which would depress the cross-correlation).

The simulated galaxy catalogs were constructed using the same selection criteria used for the real optical catalog. Maps of the fractional galaxy overdensity were created and crosscorrelated with the associated lensing convergence map.

\section{RESULTS AND DISCUSSION}

We calculate cross-power spectra between the CMB lensing maps and the maps of fractional galaxy overdensity from each of the galaxy catalogs. Given the high source density in the galaxy catalogs and the cuts designed to enhance uniformity in source selection, we expect the noise in these correlations to be dominated by the lensing reconstruction. Noise estimates are obtained by cross-correlating the galaxy maps with 50 simulated lensing maps (including realistic noise). Results are shown in Figure 3. 


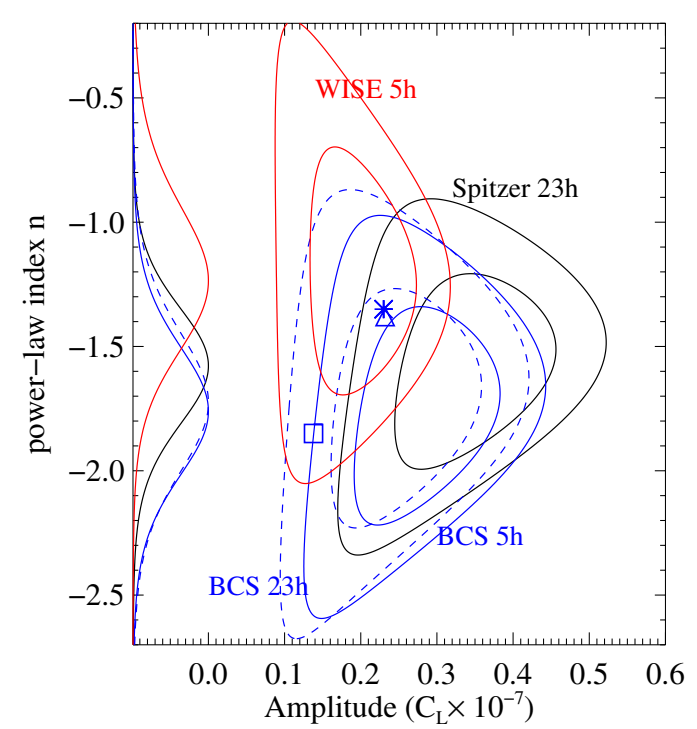

Figure 4. Power-law fits to SPT/galaxy density cross-spectra. The marginalized one-dimensional probability distributions for the power-law indices are shown projected onto the vertical axis. Also shown are the predictions from mock catalogs for optically selected galaxies (star), and predictions using the mock redshift distribution and using nonlinear (triangle) and linear (square) power spectra, assuming a bias of 1 .

(A color version of this figure is available in the online journal.)

Significant correlated signal ${ }^{38}(\sim 4.2 \sigma-5.3 \sigma)$ is detected for every catalog, and zero cross-correlation is ruled out robustly in all cases. Additionally, we note a conservative magnitude cut is used in this Spitzer analysis-extending the catalogs $1 \mathrm{mag}$ fainter increases the correlation to $>8.8 \sigma-$ but, as the properties of this deeper catalog are still being characterized, we leave detailed study of this sample to future work.

We test for contamination from Galactic cirrus by crosscorrelating lensing maps and galaxy maps with predictions for the Galactic dust emission (Finkbeiner et al. 1999) and find no correlation. We also place a limit on the systematic bias owing to tSZ using the simulated tSZ map described in Section 5. We mask an identical density of clusters as in the real CMB maps and process this map through the quadratic estimator used to create the convergence maps. The resulting maps of tSZ leakage are cross-correlated with the mock galaxy density maps. The tSZ leakage has a small effect, suppressing the correlation by $\sim 5 \%$.

A power law is fit to each measured cross-spectrum: $C_{L}=$ $A(L / 500)^{n}$. In all cases a simple power law is a good fit and the best-fit amplitudes are significantly above zero. The data are consistent with a single power law describing all data sets. The constraints on amplitude and power-law index for each crossspectrum are shown in Figure 4 and summarized in Table 1. In Figures 3 and 4, we also plot the results from the mock catalogs and convergence maps. Additionally, we plot the theoretical expectation (calculated using the CAMB software package; Lewis et al. 2000) for both the nonlinear and the linear matter power spectrum using the galaxy redshift distributions from the mock catalogs. We also constrain the redshift-independent bias assuming nonlinear evolution, by fixing the power-law index to the nonlinear value and evaluating the one-dimensional amplitude probability distribution relative to the $b=1$ value. These results are consistent with previous measurements (de la Torre et al. 2007).

${ }^{38}$ Signal-to-noise is defined as $\sqrt{\Delta \chi^{2}(0)} \cdot \Delta \chi^{2}(0)$ is the difference in $\chi^{2}$ from best fit to a model with zero cross-correlation.
The measured power-law slopes are in rough agreement with expectations for large-scale structure that is mainly in the nonlinear regime. The angular correlation function of optical galaxies is reasonably well fit by a simple power law with $w(\theta) \propto \theta^{-0.77}$ (e.g., Peebles 1975), which corresponds to $n=-1.23$. The fluctuations in the cosmic IR background are also well fit by a power law with $n=-1.2$ (Addison et al. 2012; Planck Collaboration et al. 2011; Reichardt et al. 2011). It is possible that at low $L$ the cross-correlation is probing scales that are still in the linear regime, while at high- $L$ the clustering power spectrum which dominates has a steeper slope, resulting in a good approximation of a power law. The data are not yet sufficiently constraining to test this hypothesis.

Modeling the signal further is complicated by the lack of precise redshift information for each catalog, residual contamination of the galaxy catalogs by Galactic objects, and incompleteness due to source confusion. These are not fundamental limitations, and work is ongoing to both improve and better characterize these galaxy catalogs. Even in the presence of these limitations, the cross-spectra generally appear to agree with the expectations based on both the mock catalogs and theory.

\section{CONCLUSIONS}

We have demonstrated that CMB lensing convergence maps are strongly correlated with other tracers of large-scale structure. These results are derived from a small fraction $(<10 \%)$ of the now completed $2500 \mathrm{deg}^{2}$ SPT survey, and work is underway to create convergence maps of the full survey. Realizing the full potential of the cross-correlation analyses will require deep galaxy surveys over the complete survey area. The positive correlation with WISE is promising, as all-sky WISE catalogs have recently become available. The SPT-Spitzer Deep Field will ultimately cover three times the area presented here. This same field is scheduled for observation with Herschel-SPIRE and will soon be imaged with SPTpol (Bleem et al. 2012), resulting in even deeper lensing maps. In the next few years, the Dark Energy Survey and VISTA Hemisphere Survey are expected to fully cover the SPT survey at optical and near-IR wavelengths.

With existing and planned high signal-to-noise, large-area CMB lensing maps, the near future promises rapid evolution in the state of CMB lensing analyses. Combining the lensing maps with multi-wavelength information from upcoming galaxy surveys will allow exploration of galaxy formation models out to high redshift and of structure growth through measurement of the amplitude and evolution of cosmic density fluctuations. Careful modeling of the survey galaxy populations, sources of bias in the lensing reconstructions, and theoretical expectations for the signal will allow these cross-correlation analyses to achieve their full potential.

The SPT is supported by grants ANT-0638937 and ANT0130612, with partial support provided by PHY-0114422, the Kavli Foundation, and the Moore Foundation. Work at McGill is supported by NSERC, the CRC program, and CIfAR, and at Harvard by grant AST-1009012. R. Keisler acknowledges NASA Hubble Fellowship grant HF-51275.01, B. A. Benson a KICP Fellowship, M. Dobbs an Alfred P. Sloan Research Fellowship, L. Shaw grant AST-1009811, R. Wechsler DOE contract DE-AC02-76SF00515, A. Vallinotto DOE contract DE-AC5206NA25396 (LA-UR-12-20137), and O. Zahn a BCCP fellowship. This publication uses data from the Wide-field Infrared 
Survey Explorer, a joint project of UCLA, and JPL/Caltech, funded by NASA, and uses data provided by NOAO PI: 2005B0043, distributed by the NOAO Science Archive. NOAO is operated by AURA under cooperative agreement with the NSF. This work is based in part on observations made with the Spitzer Space Telescope, operated by JPL, Caltech, under a contract with NASA.

Facilities: Blanco (MOSAIC), Spitzer (IRAC), SPT, WISE

\section{REFERENCES}

Addison, G. E., Dunkley, J., Hajian, A., et al. 2012, ApJ, 752, 120 Arnaud, M., Pratt, G. W., Piffaretti, R., et al. 2010, A\&A, 517, A92 Ashby, M. L. N., Stern, D., Brodwin, M., et al. 2009, ApJ, 701, 428

Bertin, E., \& Arnouts, S. 1996, A\&AS, 117, 393

Biesiadzinski, T., McMahon, J. J., Miller, C. J., Nord, B., \& Shaw, L. 2012, ApJ, submitted, arXiv:1201.1282

Bleem, L., Ade, P., Aird, K., et al. 2012, J. Low Temp. Phys., 167, 859

Brodwin, M., Brown, M. J. I., Ashby, M. L. N., et al. 2006, ApJ, 651, 791

Carlstrom, J. E., Ade, P. A. R., Aird, K. A., et al. 2011, PASP, 123, 568

Cooray, A., \& Hu, W. 2000, ApJ, 534, 533

Cunha, C. E., Huterer, D., Busha, M. T., \& Wechsler, R. H. 2012, MNRAS, 423, 909

Dalal, N., Doré, O., Huterer, D., \& Shirokov, A. 2008, Phys. Rev. D, 77, 123514

Das, S., Marriage, T. A., Ade, P. A. R., et al. 2011a, ApJ, 729, 62

Das, S., Sherwin, B. D., Aguirre, P., et al. 2011 b, Phys. Rev. Lett., 107, 021301

de la Torre, S., Le Fèvre, O., Arnouts, S., et al. 2007, A\&A, 475, 443

de Putter, R., Zahn, O., \& Linder, E. V. 2009, Phys. Rev. D, 79, 065033

Desai, S., Armstrong, R., Mohr, J. J., et al. 2012, ApJ, submitted, arXiv: 1204.1210
Finkbeiner, D. P., Davis, M., \& Schlegel, D. J. 1999, ApJ, 524, 867

Gerdes, D. W., Sypniewski, A. J., McKay, T. A., et al. 2010, ApJ, 715, 823

Hanson, D., Challinor, A., Efstathiou, G., \& Bielewicz, P. 2011, Phys. Rev. D, 83,043005

High, F. W., Stubbs, C. W., Rest, A., Stalder, B., \& Challis, P. 2009, AJ, 138, 110

Hilbert, S., Hartlap, J., White, S. D. M., \& Schneider, P. 2009, A\&A, 499, 31

Hirata, C. M., Ho, S., Padmanabhan, N., Seljak, U., \& Bahcall, N. A. 2008, Phys. Rev. D, 78, 043520

Hu, W., \& Okamoto, T. 2002, ApJ, 574, 566

Jeong, D., Komatsu, E., \& Jain, B. 2009, Phys. Rev. D, 80, 123527

Kaiser, N. 1992, ApJ, 388, 272

Keisler, R., Reichardt, C. L., Aird, K. A., et al. 2011, ApJ, 743, 28

Koester, B. P., McKay, T. A., Annis, J., et al. 2007, ApJ, 660, 239

Lesgourgues, J., Perotto, L., Pastor, S., \& Piat, M. 2006, Phys. Rev. D, 73, 045021

Lewis, A., Challinor, A., \& Lasenby, A. 2000, ApJ, 538, 473

Limber, D. N. 1953, ApJ, 117, 134

Miknaitis, G., Pignata, G., Rest, A., et al. 2007, ApJ, 666, 674

Peebles, P. J. E. 1975, ApJ, 196, 647

Planck Collaboration, Ade, P. A. R., Aghanim, N., et al. 2011, A\&A, 536, A18

Reichardt, C. L., Ade, P. A. R., Bock, J. J., et al. 2009, ApJ, 694, 1200

Reichardt, C. L., Shaw, L., Zahn, O., et al. 2011, ApJ, submitted, arXiv: 1111.0932

Rest, A., Stubbs, C., Becker, A. C., et al. 2005, ApJ, 634, 1103

Skrutskie, M. F., Cutri, R. M., Stiening, R., et al. 2006, AJ, 131, 1163

Smith, K. M., Zahn, O., \& Doré, O. 2007, Phys. Rev. D, 76, 043510

Song, Y.-S., Cooray, A., Knox, L., \& Zaldarriaga, M. 2003, ApJ, 590, 664

The Planck Collaboration. 2006, arXiv:astro-ph/0604069

van Engelen, A., Keisler, R., Zahn, O., et al. 2012, ApJ, submitted, arXiv: 1202.0546

Wright, E. L., Eisenhardt, P. R. M., Mainzer, A. K., et al. 2010, AJ, 140, 1868 